\section{UJMM

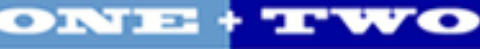

Volume 9 | 2018 Fall 2018

\section{Undergraduate Journal of Mathematical} Modeling: One + Two

2018

\title{
Comparing Two Flywheel-Piston Linkages
}

Anthony Sanchez

University of South Florida

\author{
Advisors: \\ Arcadii Grinshpan, Mathematics and Statistics \\ Scott Campbell, Chemical \& Biomedical Engineering \\ Problem Suggested By: Scott Campbell
}

Follow this and additional works at: https://digitalcommons.usf.edu/ujmm

Part of the Mathematics Commons

UJMM is an open access journal, free to authors and readers, and relies on your support: Donate Now

\section{Recommended Citation}

Sanchez, Anthony (2018) "Comparing Two Flywheel-Piston Linkages," Undergraduate Journal of Mathematical Modeling: One + Two: Vol. 9: Iss. 1, Article 4.

DOI: https://doi.org/10.5038/2326-3652.9.1.4897

Available at: https://digitalcommons.usf.edu/ujmm/vol9/iss1/4 


\title{
Comparing Two Flywheel-Piston Linkages
}

\begin{abstract}
The main idea of this paper is to compare two different ways of linking a flywheel to a piston: the Scotch Yoke linkage and the Eccentric linkage. The Scotch Yoke linkage is a way to convert the linear motion of a piston into rotational motion by using a flywheel, or vice versa. The Eccentric mechanism, on the other hand, consists of a circular wheel that is fixed to a rotating axle that makes it rotate. We compare two methods by expressing both motions as functions of three variables $(R, L$ and $\theta)$ and use these functions to illustrate the ways of linking a flywheel to a piston. Also we determine the angles $\theta$ for which the piston velocity $v$ is at its maximum.
\end{abstract}

\section{Keywords}

flywheel, piston, linear motion, rotational motion, velocity, angular velocity, cubic equation

\section{Creative Commons License}

\section{(c) (1) 99}

This work is licensed under a Creative Commons Attribution-Noncommercial-Share Alike 4.0 License. 


\section{Problem STATEMENT}

The purpose of this paper is to compare two different methods for linking a flywheel to a piston: the Scotch Yoke linkage and the Eccentric linkage. The linkages are shown in the diagram below:

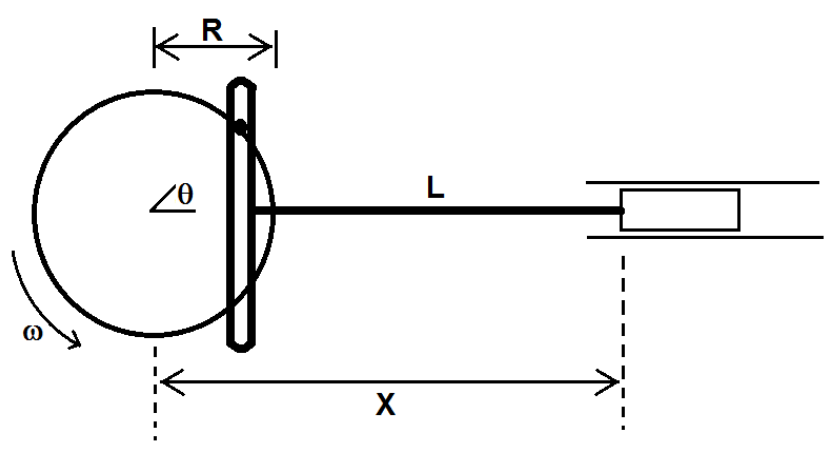

Scotch Yoke Linkage

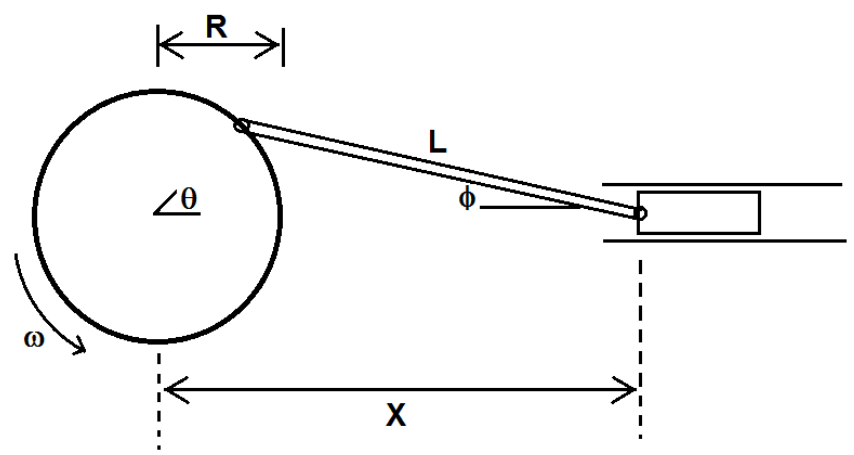

Eccentric Linkage

In both cases, the length of the linkage $L$ is $0.8 \mathrm{~m}$, the radius of the flywheel $R$ is $0.2 \mathrm{~m}$, and the flywheel rotates with a constant angular velocity $\omega=d \theta / d t=2 \pi \mathrm{rad} / \mathrm{s}$. The distance between the flywheel centerline and the piston is $x$. For both linkages we are to:

- $\quad$ express $x$ as a function of $R, L$ and $\theta$;

- develop expressions for the velocity of the piston $v=d x / d t$ as functions of $R, L$, $\theta$, and $\omega$ (plot $v$ versus $\theta$ for one revolution of the flywheel for both linkages on the same graph);

- $\quad$ use calculus to determine the angles $\theta$ for which the piston velocity $v$ is a maximum for both linkages (report the angles and the corresponding velocities); 
- discuss the behavior of the two linkages.

\section{Motivation}

Engineering has many branches that can be applied in every single aspect of life, from making food to moving people around the world by airplanes. Being an employee of a company that creates parts to power turbines made the author of the paper more interested in the work of mechanisms. In this paper, two mechanisms that make a flywheel rotate are compared in terms of their velocities and angular displacements. The first discussed method is the Scotch Yoke mechanism. It consists of joining a piston that is attached to a flywheel into a slider, and by linear motion this slider converts the energy into rotational motion making the flywheel rotate. The second method is the Eccentric mechanism. It consists of joining a rod to a piston that is fixed into an end of a flywheel, and with linear motion like in the first method rotational motion is obtained. We discuss how the maximum magnitude of their velocities can be obtained, and make a graph to compare the obtained velocities versus their angular displacements.

\section{MATHEMATICAL DESCRIPTION AND SOLUTION APPROACH}

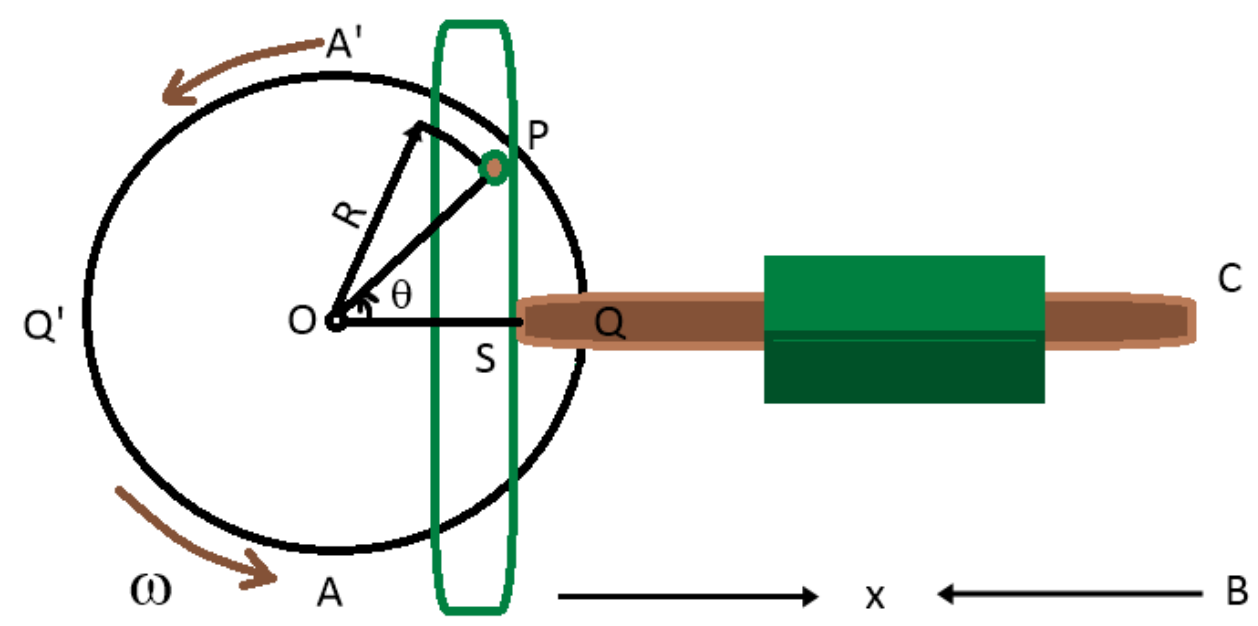


To describe a flywheel to a piston linking via the Scotch Yoke linkage, we use Figure 1 that gives a better representation of this method.

Here we appreciate how the Scotch Yoke linkage works: O represents the center of the flywheel, $\theta$ represents the angle, $R$ is the radius of the wheel. $L$ is the length of the linkage. $\mathbf{P}$ is where the pin is located and connected to the Scotch Yoke linkage mechanism. $\mathbf{S}$ is where the linkage connects the Yoke PS to the piston ( $\mathrm{S}^{\prime}$ is not shown). $\mathbf{A}$ and $\mathbf{Q}$ are positions in the wheel.

To express $x$ as a function of $R, L$ and $\theta$ we need to start working with the variables we already have:

$$
\begin{gathered}
O P=O Q=R ; \quad \angle P O Q=\theta ; S^{\prime} B=L ; \\
A B=x ; \quad A S^{\prime}+S^{\prime} B=x ; O S+L=x .
\end{gathered}
$$

By (1) we get $\cos \theta$ and $R \cos \theta$ :

$$
\cos (\angle P O S)=\frac{O S}{O P} \rightarrow \cos \theta=\frac{O S}{R} \rightarrow O S=R \cos \theta
$$

By (2) and (3) we get the following equation:

$$
x=R \cos \theta+L
$$

To know the period of the rotation of the wheel we need to understand how our variables depend on their position on the wheel. This means that when $\mathbf{S}$ is at $\mathbf{Q}, \mathbf{P}$ is at $\mathbf{Q}$; when $\mathbf{S}$ is at $\mathbf{O}, \mathbf{P}$ will be located at A'; when $\mathbf{S}$ is at $\mathbf{Q}^{\prime}, \mathbf{P}$ will be at $\mathbf{Q}^{\prime}$; when $\mathbf{S}$ goes back to $\mathbf{O}, \mathbf{P}$ will be at $\mathbf{A}$; so, when $\mathbf{S}$ goes back to $\mathbf{Q}, \mathbf{P}$ reaches $\mathbf{Q}$ again. Finally, taking into account the movement of the piston in the mentioned positions, we determine that $\mathbf{S}$ moves to $\mathbf{Q}$ and $\mathbf{Q}$ ' and repeats this motion. This means that the period is: $0 \leq \theta \leq 2 \pi$. 
Now, to calculate the velocity of the piston in terms of the angular velocity $\omega=\frac{d \theta}{d t}$ we use equation (4) that we have previously obtained:

$$
v=\frac{d x}{d t}=\frac{d}{d t}[R \cos \theta+L]=-R \frac{d \theta}{d t} \sin \theta=-R \omega \sin \theta
$$

The velocity will be positive if the rotation of the flywheel is in the counter-clockwise direction. This means that the magnitude of the velocity is $|v|$ and its equation can be represented using (5): $|v|=|-R \omega \sin \theta|=R \omega|\sin \theta|$. So, $|v| \leq R \omega$ as $|\sin \theta| \leq 1$. It follows that the maximum magnitude of the velocity is $R \omega$.

Clearly, $|\sin \theta|=1$ when $\theta=\frac{\pi}{2}$ or $\frac{3 \pi}{2}$. It means that the magnitude of the piston will be maximum when $\mathrm{P}$ is at $\mathrm{A}^{\prime}$ and $\mathrm{A}$. Also, for this linkage after using (4) and (5) and solving for $\theta$ we obtain the following equation:

$$
\left(\frac{x-L}{R}\right)^{2}+\frac{v^{2}}{R^{2} \omega^{2}}=\cos ^{2} \theta+\sin ^{2} \theta=1,
$$

that gives us the velocity of $\mathrm{P}$ in terms of $R, x, L$ and $\omega$. So, the velocity of $\mathrm{P}$ will have a maximum magnitude of $R \omega$ if $x=L$. Since $v^{2} \geq 0$ then $\rightarrow R^{2} \geq(x-L)^{2}$. It gives the solution for the Scotch Yoke linkage. 
To describe the link of a flywheel to a piston by the Eccentric linkage, we use Figure 2:

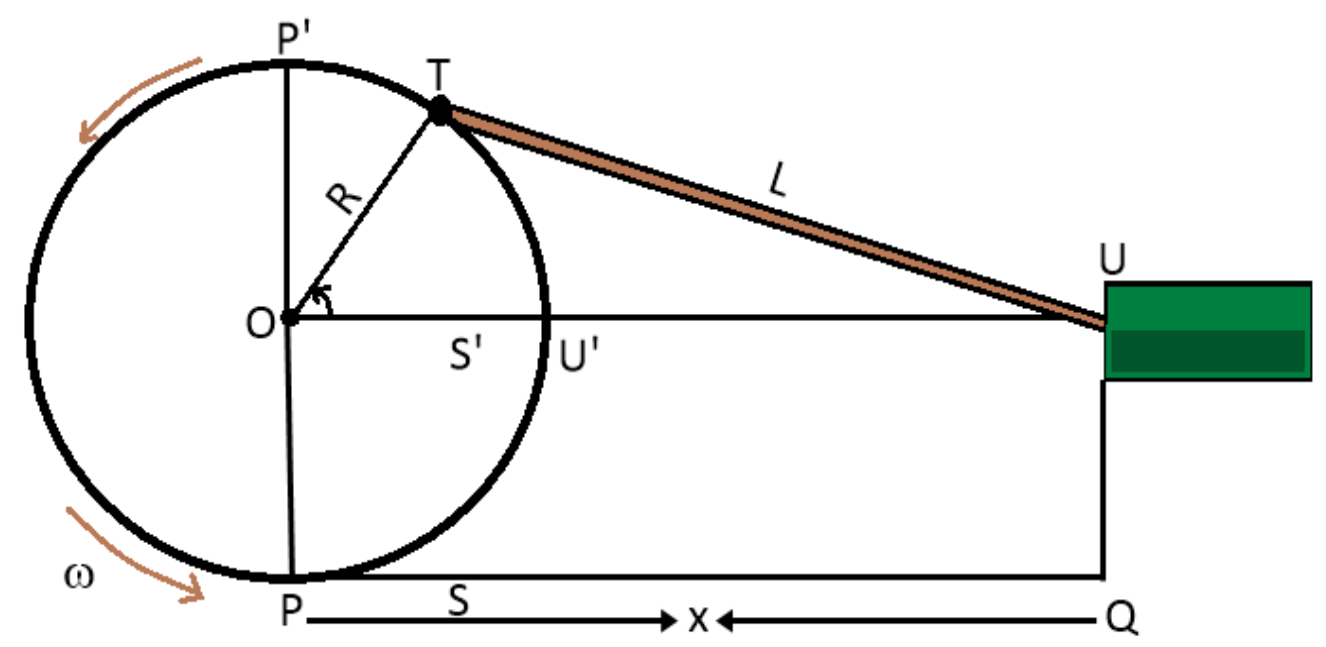

Figure 2

In Figure 2 we appreciate how the Eccentric Mechanism works:

$P Q=O U=x ; O T=O U^{\prime}=R ; T U=L$.

From (7), $x$ is the distance between the center of the wheel and the piston; $R$ is the radius of the wheel; $L$ can be represented as the length of the linkage. We also have $\mathbf{T}$, which is the connection point between the wheel and the eccentric linkage. We have the following period: $0 \leq \theta \leq 2 \pi$. So,

$$
\angle \operatorname{TOS}^{\prime}=\theta \text {. }
$$

We are to use the known formulas:

$\sin 2 \theta=2 \sin \theta \cos \theta ; \cos 2 \theta=1-2 \sin ^{2} \theta$.

By (7) and (8), we obtain:

$$
x=O U=O S^{\prime}+S^{\prime} U=O T \cos \theta+S^{\prime} U
$$


$\rightarrow x=R \cos \theta+\sqrt{T U^{2}-S^{\prime} T^{2}}=R \cos \theta+\sqrt{L^{2}-S^{\prime} T^{2}}$

$\rightarrow x=R \cos \theta+\sqrt{L^{2}-\left(O T^{2}-S^{\prime} O^{2}\right)}$

$\rightarrow x=R \cos \theta+\sqrt{L^{2}-\left(R^{2}-(R \cos \theta)^{2}\right)}$

$\rightarrow x=R \cos \theta+\sqrt{L^{2}-R^{2} \sin ^{2} \theta}$.

Also (9) implies that:

$(x-R \cos \theta)^{2}=L^{2}-R^{2} \sin ^{2} \theta$

$\rightarrow x^{2}+R^{2}-2 x R \cos \theta-L^{2}=0$.

To calculate velocity in terms of the angular velocity $\omega=\frac{d \theta}{d t}$, we use:

$$
\begin{aligned}
& v=\frac{d x}{d t}=\frac{d}{d t}\left[R \cos \theta+\sqrt{L^{2}-R^{2} \sin ^{2} \theta}\right] \\
& \rightarrow=-R \sin \theta \frac{d \theta}{d t}-\frac{R^{2} \sin \theta \cos \theta}{\sqrt{L^{2}-R^{2} \sin ^{2} \theta}} \frac{d \theta}{d t} \\
& \rightarrow=-R \omega \sin \theta-\frac{R^{2} \sin \theta \cos \theta}{\sqrt{L^{2}-R^{2} \sin ^{2} \theta}} \omega ; \\
& \rightarrow v=-R \omega \sin \theta-\frac{R^{2} \omega \sin 2 \theta}{2 \sqrt{L^{2}-R^{2} \sin ^{2} \theta}} .
\end{aligned}
$$

From (10), we see that the velocity $v$ exists when:

$$
L^{2}-R^{2} \sin ^{2} \theta>0 \rightarrow \text { This means that } \rightarrow \sin ^{2} \theta<\frac{L^{2}}{R^{2}} .
$$

From equations (9) and (10) we obtain:

$$
\begin{aligned}
& \rightarrow v=-R \omega \sin \theta-\frac{R^{2} \omega \sin 2 \theta}{2(x-R \cos \theta)} \\
& \rightarrow v=-\frac{x R \omega \sin \theta}{(x-R \cos \theta)}
\end{aligned}
$$

Our velocity $v$ exists when $x-R \cos \theta>0 \rightarrow \cos \theta<\frac{x}{R}$. 
It means that the Eccentric linkage for joining a flywheel to a piston is possible when

$$
\cos \theta<\frac{x}{R} \text { and } \sin ^{2} \theta<\frac{L^{2}}{R^{2}}
$$

Our values will be positive if the flywheel is rotating in the counterclockwise direction, and our velocity $v$ will be at its maximum when we have: $\frac{d v}{d \theta}=0$. Hence:

$\rightarrow-R \omega \cos \theta-R^{2} \omega\left[\frac{R^{2} \sin ^{4} \theta+L^{2} \cos (2 \theta)}{\left(L^{2}-R^{2} \sin ^{2} \theta\right)^{\frac{3}{2}}}\right]=0$.

Equation (11) implies that:

$$
\begin{gathered}
\cos \theta\left(L^{2}-R^{2} \sin ^{2} \theta\right)^{\frac{3}{2}}=-R\left[R^{2} \sin ^{4} \theta+L^{2} \cos (2 \theta)\right] \\
\rightarrow\left(\cos ^{2} \theta\right)\left(L^{2}-R^{2} \sin ^{2} \theta\right)^{3}=R^{2}\left[R^{2} \sin ^{4} \theta+L^{2} \cos (2 \theta)\right]^{2}
\end{gathered}
$$

Then we square both sides and obtain the following equation:

$R^{6} \sin ^{6} \theta-L^{2} R^{4} \sin ^{4} \theta\left(1+\sin ^{2} \theta\right)+L^{4} R^{2}\left(1+\sin ^{4} \theta-\sin ^{2} \theta\right)-L^{6}\left(1-\sin ^{2} \theta\right)=0$, that can be reduced to equation (12) via dividing it by $\left(1-\frac{L^{2}}{R^{2}}\right)$ if $L \neq R$ :

$$
R^{6} \sin ^{6} \theta-L^{2} R^{4} \sin ^{4} \theta-L^{4} R^{2} \sin ^{2} \theta+L^{4} R^{2}=0
$$

Therefore, the velocity $v$ of the piston connected to the flywheel using the Eccentric linkage reaches its maximum magnitude when a value of $\theta$ satisfies equation (12). The resulting equation (12) can be solved by replacing $\sin ^{2} \theta=y$, and doing this we get:

$$
R^{6} y^{3}-L^{2} R^{4} y^{2}-L^{4} R^{2} y+L^{4} R^{2}=0 .
$$

Our resulting equation (13) is a cubic equation in terms of $y$, so it has at least one real root. The equation can be solved to get the proper value of $y$, and then replaced back to $\sin ^{2} \theta$ as above, so we can eventually obtain our value for $\theta$.

Now, after determining the velocities $v$ of the piston by using the Scotch Yoke and Eccentric linkages, we can compare them versus the angular displacements $\theta$. 
For one revolution of the wheel using $v$ versus $\theta$, and our given values in the problem statement we have:

$L=0.8=\frac{4}{5} m \quad ; \quad R=0.2=\frac{1}{5} m ; \omega=2 \pi \frac{\mathrm{rad}}{\mathrm{s}}$

Now, since we compare the velocities of two linkages let us denote them as $V_{1}$ (Scotch Yoke linkage) and $V_{2}$ (Eccentric linkage). By equations (5), (10), and using values (14) we obtain:

$V_{1}=-\frac{2 \pi \sin \theta}{5} ; V_{2}=-\frac{2 \pi \sin \theta}{5}-\frac{\pi \sin 2 \theta}{5 \sqrt{16-\sin ^{2} \theta}}$

For our graph, we need to plot the following values for $\theta$ that correspond to one revolution of the flywheel:

$\theta=0, \frac{\pi}{4}, \frac{\pi}{2}, \frac{3 \pi}{4}, \pi, \frac{5 \pi}{4}, \frac{3 \pi}{2}, \frac{7 \pi}{4}, 2 \pi$.

Finally, by using our equations and values (14), (15), and (16) we can graph velocities $V_{1}$ and $V_{2}$ versus their angular displacements $\theta$ when they meet the same condition in terms of the given values for $L, R$ and $\omega$. 


\section{Piston Velocities (v) versus Angular Displacements $(\theta)$}

Velocity of the Scotch Yoke Linkage

Velocity of the Eccentric Linkage

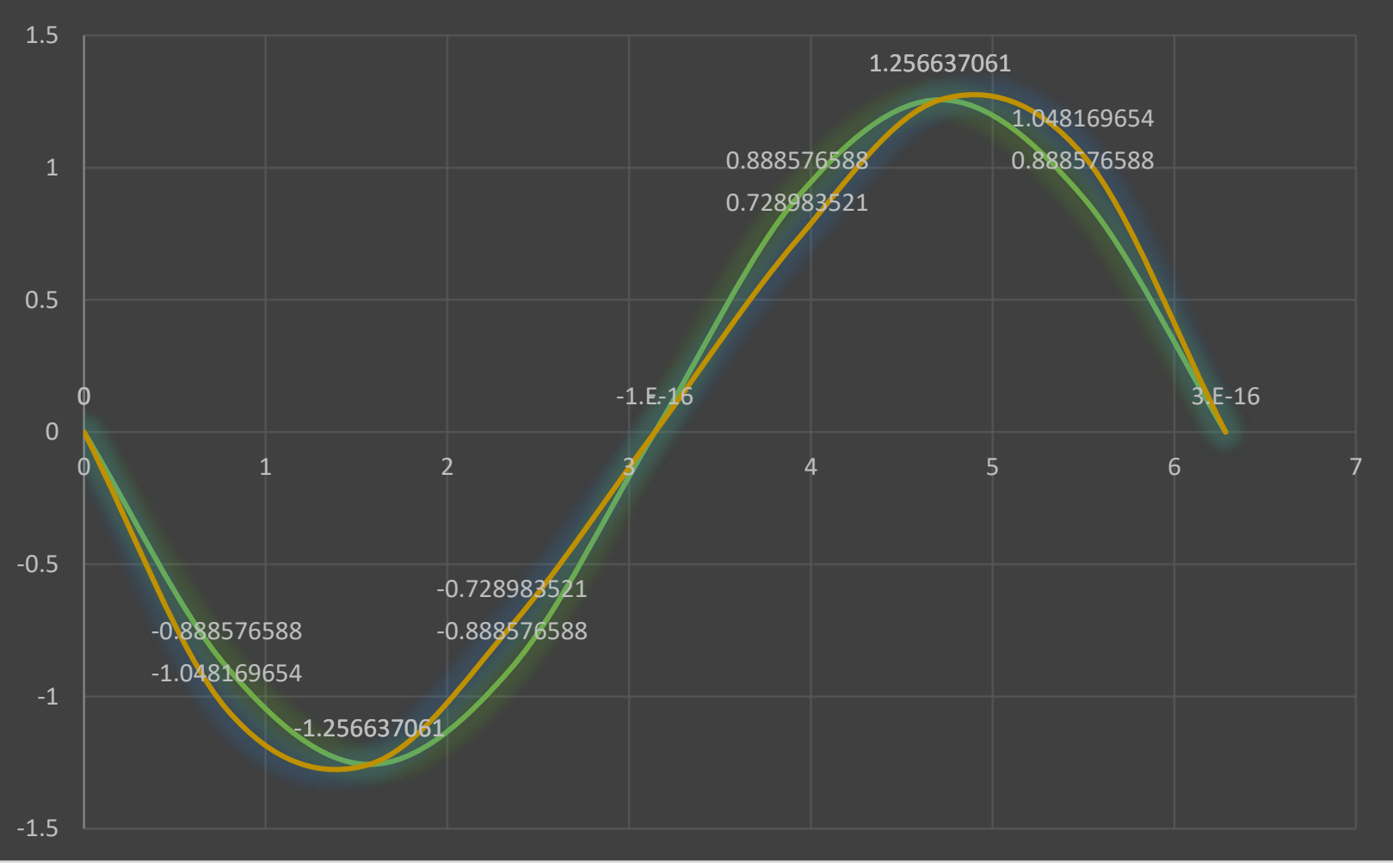

Figure 3

For Figure 3 we use equation (15) and plot the values (16) to obtain the given results for the graph $\left[V_{1}\right.$ (Scotch Yoke linkage) and $V_{2}$ (Eccentric linkage) $]$. The results are based on one complete revolution: $0 \leq \theta \leq 2 \pi$.

\section{DISCUSSION}

- From the obtained graph, we can determine that the velocities

$V_{1}$ (Scotch Yoke linkage) and $V_{2}$ (Eccentric linkage) are sinusoids.

Also we can observe the following aspects:

- for $0 \leq \theta \leq \frac{\pi}{2}$ and $\frac{3 \pi}{2} \leq \theta \leq 2 \pi \rightarrow\left|V_{2}\right| \geq\left|V_{1}\right|$ 
- for $\frac{\pi}{2} \leq \theta \leq \pi$ and $\pi \leq \theta \leq \frac{3 \pi}{2} \rightarrow\left|V_{2}\right| \leq\left|V_{1}\right|$

- at $\theta=0, \pi, 2 \pi \rightarrow\left|V_{2}\right|=\left|V_{1}\right|$

- We obtain the maximum magnitude of the Scotch Yoke linkage $\left(V_{1}\right)$ when $\theta=\frac{\pi}{2}$ and $\frac{3 \pi}{2}$.

- We observe that the velocities from $V_{1}$ and $V_{2}$ at $0 \leq \theta \leq \pi$ are very similar in their shape since they both are sinusoidal. However, they have opposite signs when $\pi \leq \theta \leq 2 \pi$.

- We observe that in both cases the pistons move at the same rate, and along same plane in the $x$-axis.

- We obtain the maximum value of the Eccentric linkage $\left(V_{2}\right)$ when

$$
\theta \in\left(\frac{\pi}{4}, \frac{\pi}{2}\right) \text { and } \theta \in\left(\frac{3 \pi}{2}, \frac{7 \pi}{4}\right) \text {. }
$$

Now, to obtain the maximum magnitude we need to use the previously obtained equation (12), which is equivalent to the cubic equation (13) that has at least one real root. We need to use values (14) in equations (12) and (13) to obtain the following result:

$$
\begin{aligned}
& \sin ^{6} \theta-16 \sin ^{4} \theta-256 \sin ^{2} \theta+256=0 \\
& \rightarrow y^{3}-16 y^{2}-256 y+256=0, \\
& \text { where } y=\sin ^{2} \theta .
\end{aligned}
$$

Having used WolframAlpha we get the following roots of this cubic equation: $y \approx 25.607,-10.554,0.94724$. Since $0 \leq \sin ^{2} \theta \leq 1$ we have that $0 \leq y \leq 1$. Hence we can discard $y \approx 25.607$ and -10.554 choosing $y \approx 0.94724$ that gives us $\sin ^{2} \theta \approx 0.94724$. By root-squaring both sides we obtain $\sin \theta \approx \pm 0.973263$. A more detailed calculation gives $\sin \theta \approx \pm 0.973263036$.

Converting our result to degrees we obtain: $\theta \approx 76.72097779^{\circ}, 103.2790222^{\circ}, 256.7209778^{\circ}, 283.2790222^{\circ}$

Now, by equation (14) for plotting these values we obtain:

$$
\begin{array}{ll}
\theta \approx 76.72097779^{\circ} & \left|V_{2}\right| \approx 1.295445405 \\
\theta \approx 103.2790222^{\circ} & \left|V_{2}\right| \approx 1.150631398 \\
\theta \approx 256.7209778^{\circ} & \left|V_{2}\right| \approx 1.150631398
\end{array}
$$




$$
\theta \approx 283.2790222^{\circ} \quad\left|V_{2}\right| \approx 1.295445405
$$

Clearly, the maximum magnitude of the velocity $v$ for the Eccentric linkage $\left(V_{2}\right)$ is possible when the angular displacements $\theta$ are at:

$\theta \approx 76.72097779^{\circ}$ and $283.2790222^{\circ}$ so we can confirm that our values for $\theta \in\left(\frac{\pi}{4}, \frac{\pi}{2}\right)$ and $\theta \in\left(\frac{3 \pi}{2}, \frac{7 \pi}{4}\right)$ are true.

Given these results, we can compare our graph (Figure 3) with the derivations we have done to solve for the Eccentric linkage, and we can determine that the values agree for both the graph and mathematical approach making our problem solvable.

\section{CONCLUSION AND RECOMMENDATIONS}

The comparison between the linkages of a flywheel to a piston by the Scotch Yoke linkage and the Eccentric method is done. By using calculus, we obtain the values of their velocities $(v)$ and specified angular displacements $(\theta)$ when they reach their maximum magnitude. Also, by having the values of the angular displacement and using the equations that are obtained in the paper we present a graph (Figure 3) that shows the velocities $(v)$ versus the angular displacement $(\theta)$ for a complete revolution of the rotational motion for: $V_{1}$ (Scotch Yoke linkage) and $V_{2}$ (Eccentric linkage).

In addition, a recommendation would be first to understand the problem, then state and solve for the variables until the solution is found. After a set of equations that allow us to solve the considered problem is set up the given values should be used. This recommendation is to avoid big numbers in the beginning and save time during mathematical analysis since people can get confused if the values are plugged into the first equations that are found. 


\section{NOMENCLATURE}

Symbol

$v$

$L$

$V_{1}$

$V_{2}$

$R$

$\theta$

$\omega$
Description

Value

$m / s$

$m$

Length of the linkage

$m / s$

Velocity of the Scotch Yoke linkage

$m / s$

Velocity of the Eccentric linkage

radius of the flywheel

$m$

$\circ$

angles

angular velocity $\mathrm{rad} / \mathrm{s}$

\section{REFERENCES}

Chegg Study, Question: Comparison of two flywheel-piston linkages,

https://www.chegg.com/homework-help/questions-and-answers/comparison-two-flywheel-

piston-linkages-purpose-project-compare-two-different-methods-link-q25139005

Animated Mechanisms, http://www.mekanizmalar.com/index.html, Scotch Yoke,

http://www.mekanizmalar.com/scotch_yoke.html

Food Technology Lectures. YouTube, 287 Slider crank mechanism with eccentric.

Online video clip, https://www.youtube.com/watch?v=b0gQyBgGSHY .

Vinod S. Achary, Scotch Yoke Mechanism *Most viewed Scotch Yoke animation on youtube*. Online video clip. YouTube, https://www.youtube.com/watch?v=hsaoTo1vuY4 .

James Stewart, Essential calculus: Early Transcendentals, 2nd Edition. Belmont, CA:

Brooks/Cole, Cengage Learning, 2013. 